\title{
Mechanical vibrations in the transport of hatching eggs and the losses caused in the hatch and quality of broiler chicks
}

\author{
Ana Carolina Donofre, ${ }^{1}$ Iran José Oliveira Silva, ${ }^{1}$ Aérica Cirqueira Nazazerno, ${ }^{1}$ \\ Iuri Emmanuel De Paula Ferreira ${ }^{2}$
}

${ }^{1}$ Environment Research Unit, Department of Biosystem Engineering, University of São Paulo; ${ }^{2}$ Center of Natural Sciences, Federal University of São Carlos, Brazil

\begin{abstract}
Many factors present in eggs' transportation from incubator to growing confinements can damage their integrity and, consequently, the results of the incubation. This study aimed to evaluate whether the intensity and duration of exposures of hatching eggs to mechanical vibrations were capable of affecting the hatch and the quality of broiler chicks. Four treatments, determined by two intensities of vibrations and two durations of exposures were evaluated and compared with a control group in a randomised design in blocks with four repetitions. The vibrations were applied by a mechanical shaker before incubation. Then the eggs were incubated and as the following responses were evaluated: hatchability, the type of embryonic mortality, the quality of the birds and the total of losses. The analyses were carried out by a binomial regression model, with application of the Wald test at 5\% significance level. The results showed that the exposure of eggs to mechanical vibrations harmfully affects the hatchability and reduces the ratio (percentage) of high quality chicks. Highest vibration level together with longer duration of exposure resulted in worst hatchability and losses. It is argued that the mechanical vibrations are potentially damaging and should be regarded as an important factor in the management of hatching eggs.
\end{abstract}

Correspondence: Ana Carolina Donofre, Environment Research Unit, Department of Biosystem Engineering, University of São Paulo, Av. Padua Dias 11, P.O. Box 9, zip code 13418-900, Piracicaba, SP, Brazil. Tel.: +55.019.3447.8563.

E-mail: acdonofre@usp.br

Key words: Broiler production; hatchability; hatchery; resultant acceleration.

Received for publication: 18 August 2016.

Accepted for publication: 17 October 2016

(C) Copyright A.C. Donofre et al., 2017

Licensee PAGEPress, Italy

Journal of Agricultural Engineering 2017; XLVIII:593

doi:10.4081/jae.2017.593

This article is distributed under the terms of the Creative Commons Attribution Noncommercial License (by-nc 4.0) which permits any noncommercial use, distribution, and reproduction in any medium, provided the original author(s) and source are credited.

\section{Introduction}

All procedures involving the manipulation of hatching eggs have their relevance in the hatch and quality of broiler chicks and the logistics of live load in Brazil, specifically in the aviculture sector, faces various problems (Nazareno et al., 2015). In this study, attention is drawn to the multifactorial problem of road transport, focused on mechanical vibrations. Would these movements capable of compromising the physical integrity of eggs and, consequently, the results of incubation?

The material transported is constantly exposed to vibrations, which, in their majority, are harmful to the same. By leveraging these effects, the intensity of vibratory movements depends on factors related to vehicles, such as the suspension system and the number of axles. There is also a strong influence by roads' conditions, driver's performance and provision of loads (Gebresenbet et al., 2011).

Several authors argue that the mechanical vibrations negatively influence the poultry chain. The main consequences of this agent are related to stress and a drop in quality of broilers' meat transported for slaughter (Randall et al., 1993; Randall et al., 1997; Carlisle et al., 1998; Abeyesinghe et al., 2001), in addition to the loss of eggs for human consumption and/or hatching, as a result of cracks, breaks and changes in internal quality (Berardinelli et al., 2003a, 2003b; Torma and Kovácsné, 2012; Nazareno et al., 2013).

The integrity of eggshell, yolk and albumen is also likely to change, given the exposure of eggs to mechanical vibrations. The results were related to the cracks in eggshells, liquefaction of albumen with reduction in the values of Haugh unit and the lower resistance of the yolk's membrane, clearly demonstrating the negative effects of vibrations on the internal quality of eggs (Berardinelli et al., 2003a, 2003b). But, what would happen with hatching eggs get exposed to vibrations? Few tests were conducted and the results still need confirmations. To prove that the exposure to vibration may impair the development of the embryo, Shannon et al. (1994) daily throbbed hatching eggs during incubation, with frequencies of 5 to $50 \mathrm{~Hz}$ and acceleration RMS of up to $5 \mathrm{~g}$. The main evidence was an increase of $32 \%$ in embryonic mortality. Torma and Kovácsné (2012) also found a reduction of approximately $15 \%$ in hatchability in treatment with intense vibration compared to a control treatment.

We want to prove the harmful effects of mechanical vibration of transport. So, this study aimed to evaluate whether the exposure of hatching eggs to mechanical vibrations can also compromise the hatch and the quality of broiler chicks. 


\section{Materials and methods}

\section{Simulations and characterisation of mechanical vibration}

The vibrations during transport were simulated by a mechanical shaker capable of producing periodic movements with a frequency of $20.8 \mathrm{~Hz}$ in three orthogonal axes (x,y, and z), by an engine of eccentric rotation, connected to a support platform that represents the truck's floor. Vibrations produced by the shaker engine were transmitted by the shelter anisotropically through a system of elastic and spring tensioners, in such a way the vertical movements were more intense, simulating the response of trucks due to bumps and holes on the roads during transportation. To describe the intensity of vibration factor, the movements were monitored by an HOBO Pendant G UA-004- $64^{\circledR}$ accelerometer, with measurement range of $\pm 3 \mathrm{~g}$ or $29.4 \mathrm{~m} \mathrm{~s}^{-2}$. The equipment was coupled to the simulation system, to which later could be obtained the resultant acceleration (RSS; $\mathrm{m} \mathrm{s}^{-2}$ ).

The RSS is often used to quantify the intensity of the mechanical vibration. This measurement is represented by a single value, obtained from the square root of the sum of the squares of the resultant acceleration (RMS; $\mathrm{m} \mathrm{s}^{-2}$ ) for each of the axes, as well as denoted in equations 1 and 2 (Gebresenbet et al., 2011; Nazareno et al., 2013):

$$
\mathrm{RMS}_{\mathrm{j}}=\left(\frac{\sum_{\mathrm{t}} \mathrm{a}\left(\mathrm{t}_{\mathrm{j}}^{2}\right.}{\mathrm{N}}\right)^{1 / 2}
$$

where:

$a_{j}(t)$ is the observed value of instant acceleration on axe $\mathrm{j}$ (x, y or $\mathrm{z}$ ) and at time $\mathrm{t}(\mathrm{t}=1,2, \ldots, \mathrm{N}) ; \mathrm{N}$ is the total number of observations in the respective axis;

$$
\mathrm{RSS}=\left(\mathrm{RMS}_{\mathrm{x}}^{2}+\mathrm{RMS}_{\mathrm{y}}^{2}+\mathrm{RMS}_{\mathrm{z}}^{2}\right)^{1 / 2}
$$

The RMS of $\mathrm{x}, \mathrm{y}$ and $\mathrm{z}$ (Eq. 1) was determined by the values of instant acceleration $\left(\mathrm{m} \mathrm{s}^{-2}\right)$, collected by accelerometers every ten seconds during the simulations, so that could be calculated the RSS used as the reference value of the treatments (RSS 2.5 or 7.5 $\mathrm{m} \mathrm{s}^{-2}$ ). The maximum shock reached up to 5 or $10 \mathrm{~m} \mathrm{~s}^{-2}$ (depending on the vibration level that was used) in the $y$ axis, while only half of these values were verified for $\mathrm{x}$ and $\mathrm{z}$ axes.

\section{Experimental procedures}

The experimental design was randomised in blocks, with four repetitions, in a $2 \times 2+1$ factorial scheme (Bailey and Łacka, 2015). The factorial was determined by two intensities of vibration, two times of exposure to vibrations (minimum and maximum) and a control treatment. The intensity of lower vibration was characterised by RSS de $2.5 \mathrm{~m} \mathrm{~s}^{-2}$, chosen on the basis of exposure limits established by Randall et al. (1993) and Randall et al. (1997). While the higher intensity was determined by RSS de 7.5 $\mathrm{m} \mathrm{s}^{-2}$, to simulate the data of Nazareno et al. (2013) found in real conditions.

The time of exposure factor included a minimum period of 60 min and a maximum of 180 minutes, which accounted for two transport situations: short and medium distance. The variations in the vibration intensity and exposure time formed the treatments of factorial part T1 (lower vibration; minimum time), T2 (lower vibration; maximum time), T3 (upper vibration; minimum time) and T4 (upper vibration; maximum time). Recalling that, in addition to the factorial treatments, there was an additional treatment represented by eggs not exposed to vibrations (control).

The simulations were conducted in a commercial hatchery, so that incubation could be performed after the application of the treatments, as well as to ensure the control of other factors, which were not experimental. The used eggs came from the same batch of arrays of Cobb 500, the age of the birds ranged from 32 to 35 weeks, in the course of four repetitions (blocks) of the experiment. For the parcels' assembling, the eggs were selected (disposal of broken, cracked or defective) and rearranged in plastic trays. By repetition, 96 eggs were used in each treatment. The set of trays was placed in a plastic box positioned on the shaker, simulating the system box of eggs - vehicle's floor. The accelerometer was horizontally fixed on one side of the plastic box and additional trays full of eggs were used in order to completing the total capacity of the unit (240 eggs), as in fact occurs in transport.

After the simulations, the five treatments came at the same time to the incubator (CASP, CMG 125e), being held on the same incubation conditions by approximately $504 \mathrm{~h}$, when they were removed once for the registration of the variable responses of interest. The repetitions occurred in four consecutive weeks, whose differences were controlled for the effect of blocks. The time and the conditions of storage until the beginning of the simulations were standardised, as well as the temperature and the relative humidity $\left(17-18^{\circ} \mathrm{C}\right.$ and $67-75 \%$, respectively), which were maintained constant during simulations and storage of eggs.

\section{Response variables}

It was evaluated the hatchability, determined by the ratio of birds hatched alive by all of incubated fertile hatching eggs. For distinction of fertile unhatched eggs from infertile eggs was conducted a break of them and analysis of the waste.

With the analysis of residues, it was also identified the type of embryonic mortality, i.e. the period in which the embryo had its development interrupted because of failure of its system. In this response, mortality was considered early dead embryos that seemed to be between $1^{\text {th }}$ and $5^{\text {th }}$ day of development; intermediate from the $6^{\text {th }}$ to $17^{\text {th }}$ day; and late from 18 to 21 days. The pecked eggs, when the birds have pierced the shell but not left, entered the classification as late mortality.

In assessing the quality of birds newly hatched, they were separated into two groups: the high quality chicks (first line) and low quality chicks (second line). For this, it was used the criteria presented in Table 1.

Finally, total loss was calculated by summing the number of unhatched eggs and chicks with low quality, which supposed to be discarded at the hatchery.

\section{Statistical analysis}

The responses hatchability, type of mortality, high quality chicks and total losses were analysed by a generalised linear model, using the binomial distribution with the logistic link function and the linear predictor expressed by:

$$
\log _{e}\left(\frac{\pi_{i k}}{1-\pi_{i k}}\right)=\mu+\delta_{k}+\tau_{i}
$$

where:

$\pi_{\mathrm{ik}}$ is the proportion of hatching eggs in the tray corresponding to 
the i-th treatment of the k-th block that shows the event of interest (such as hatch, mortality, high quality chick or hatching egg loss).

In the binomial regression, they were assessed the effects of experimental factors on the natural logarithm of odds. In the model, the parameter $\mu$ determines an overall average of this logarithm, while parameters $\delta_{\mathrm{k}}$ and $\tau_{\mathrm{i}}$ indicate the effects of the k-th block and the i-th treatment, respectively. The results of the binomial regression are presented by odds and odds ratios of the event of interest. The odds ratios are determined by dividing the odds of the event of interest in a factorial treatment $(\mathrm{T} 1, \mathrm{~T} 2, \mathrm{~T} 3$ or $\mathrm{T} 4)$ by its odds in the control (intercept).

The statistical assumptions were verified from the analysis of residual deviance and by Chi-square test at 5\% level of significance, graphics components of deviation and envelopes simulated with $95 \%$ confidence for the same. The significance of the fitted models' coefficients was obtained by using the Wald test at $5 \%$ level. In addition, they were constructed intervals with $95 \%$ confidence for the odds ratios estimated. All statistical were carried out by R program (R Development Core Team, 2005).

\section{Results and discussion}

The responses of hatchability are presented in Table 2. The overall average observed in the experiment was $92.73 \%$. The odd of hatching of a fertile egg in the intercept (control in block 1) was 17.30, that is, for each 17.30 fertile eggs hatched in the control treatment, only one egg did not hatch.

Using the Wald test, at 5\% significance, it was observed that control's log odds differed only from factorial T4's, which the odds of hatching was almost half of control's $(\mathrm{CR}=0.47)$. Still, in the treatment of intense vibration (T4), it can be stated that one out of 8.13 hatched hatching eggs did not break out, confirming that the hatchability average of $88.92 \%$ is actually lower. They have not been proved isolated effects of range of vibration and exposure time in factors' unfolding for the hatchability.

None of the treatments or effects of experimental factors was able to explain the odds of occurrence of early mortality (1 to 5 days). In other words, at this stage the mortality is the same when comparing the different factor loadings with the control. Whereas the odds of an embryo dies in intermediate period (6 to 17 days) was significantly different between $\mathrm{T} 4$ and the control using the Wald test at 5\% significance level. So, the factorial showed almost two times more likely to show a dead embryo in this period $[\mathrm{CR}=2.86$; confidence interval $(\mathrm{CI})=1.08 ; 8.94]$.

In late mortality, which occurs between the $17^{\text {th }}$ and the $21^{\text {th }}$ day, including eggs pecked alive and dead, only T2 (RSS $2.5 \mathrm{~m} \mathrm{~s}^{-2} ; 180$ $\mathrm{min}$ ) differed from the control group by Wald test at a significance level of $5 \%$, whose odds ratio was only $0.12(\mathrm{CI}=0.01 ; 0.68)$, then, below the reference treatment and even at the normal rates expected. For the type of mortality, the experimental factors are not able to explain the results obtained.

In the control group, one out of 16.96 high quality chicks was classified as low quality. Result that at the end provides an average of $88.75 \%$ of high quality chicks, which would be fully used by hatchery facilities, as shown in Table 3 .

Among the comparisons of factorial treatments with the control for response high quality chicks, it was found that treatments T3 and T4 significantly differed from control at 5\% level of significance. In T3 (RSS $7.5 \mathrm{~m} \mathrm{~s}^{-2} ; 60 \mathrm{~min}$ ), one out of 9.66-hatched birds is of low quality, which determines an odds ratio of 0.57 in

Table 1. Criteria for classification of chicks in the first or second line.

\begin{tabular}{ll}
\hline High-quality chicks & Low-quality chicks \\
Absence of physical defects & Presence of physical defects, i.e., duplication of members, exposed brain, exposed viscera \\
Clean, dry, without waste of hatching in your body or skin lesions & Dirty, wet, with residues of hatching or skin lesions \\
\hline Normal beaks and eyes & Absence of eyes and beaks, broken beaks, bleeding \\
Normal members' conformation & Duplicated members, compromised or bent \\
\hline Closed umbilicus, absence of yolk sac and residual membranes & Open and darken umbilicus, presence of yolk sac and residual membranes \\
Normal size & Scrap, small or weak \\
\hline
\end{tabular}

Table 2. Mean and standard deviation of hatchability (in percentages), odds of hatching, and odds ratios between the factorial treatments and control with $\mathbf{9 5 \%}$ confidence intervals.

\begin{tabular}{lcccc} 
Treatments & Hatchability (\%) & Odds of hatching & Odds ratios & Confidence intervals \\
Control* & $94.47+1.76$ & & & 1.00 \\
Block 1 & - & 17.30 & 1.25 & $(0.74 ; 2.10)$ \\
\hline Block 2 & - & 21.54 & 0.70 & $(0.44 ; 1.11)$ \\
Block 3 & - & 12.15 & 1.18 & $(0.71 ; 2.00)$ \\
\hline Block 4 & - & 20.55 & & $(0.47 ; 1.59)$ \\
Factorial treatments & & & 0.87 & $(0.52 ; 1.85)$ \\
T1 & $93.73+2.25$ & 15.05 & 0.99 & $(0.37 ; 1.21)$ \\
T2 & $94.43+1.08$ & 17.12 & 0.68 & $(0.26 ; 0.80)$ \\
T3 & $92.12+4.67$ & 11.76 & 0.47 & \\
T4 & $88.92+2.78$ & 8.13 & & \\
\hline
\end{tabular}

${ }^{*}$ Control treatment in block 1 was considered as the baseline for odds ratios comparisons; ${ }^{\circ}$ significant effects using the Wald test at $5 \%$ level of significance. 
relation to the control group. In other words, only $57 \%$ of birds would be of high quality chicks in this treatment. The treatment $\mathrm{T} 4$, determined by upper vibration during maximum time, resulted in a proportion of hatches of high quality chicks even lower than $\mathrm{T} 3$, once one out of 6.10 hatched birds is of low quality. This result is related to an odds ratio of 0.36 , which represents a probability of occurrence of high quality chicks quite below the control (only $36 \%)$.

During effects' unfolding, the intensity of vibration showed laterally significant, at level of $5 \%$, using the Wald test $(\mathrm{CH}=0.62$; $\mathrm{CI}=0.29 ; 1.27)$. The odds to produce a high quality chick under upper vibration (RSS $7.5 \mathrm{~m} \mathrm{~s}^{-2}$ ) were only $62 \%$ as compared with lower intensity (RSS $2.5 \mathrm{~m} \mathrm{~s}^{-2}$ ).

Summing up the hatching eggs which have not hatched with low quality chicks, it was obtained the total losses response. For the control, it was observed a relation of six losses for each 100 hatching eggs. In treatments T1 e T2, the results are similar to control group, which is proven by nullity of the effects and treatments in all the previously presented responses. However, the factorial treatments $\mathrm{T} 3$ and $\mathrm{T} 4$ showed significant differences in relation to the control group (Table 4).

The results demonstrate an additional loss to treatments $\mathrm{T} 3$ and $\mathrm{T} 4$, which is certainly associated with exposure to intense vibration (RSS $7.5 \mathrm{~m} \mathrm{~s}^{-2}$ ). It was found that the odds of losses of all hatching eggs incubated in T3 and T4 was 10\% and $16 \%$, respectively. With the treatments' unfolding, the vibration severity was significant using the Wald test at 5\%, so that the odds of any loss in the upper range is up to $68 \%$ greater than the opposite range $(\mathrm{RC}=1.88$; $\mathrm{CI}=1.00 ; 2.86)$.

The total losses response showed that the combination of range and time of vibration in T3 and T4 has contributed significantly to the prejudice of those companies. In T4 (RSS $7.5 \mathrm{~m} \mathrm{~s}^{-2}$; $180 \mathrm{~min}$ ) this result was quite relevant, because it represented the odds of $10 \%$ more losses than in the control, whose proportion was only $6 \%$. Regarding to T3 (RSS $7.5 \mathrm{~m} \mathrm{~s}^{-2}$; $60 \mathrm{~min}$ ), these losses were $4 \%$ higher than the control, also significant, though.

Many studies indicate that the hatch and the quality of broiler chicks are influenced by factors such as chickens' age and storage and transport conditions of hatching eggs (Tona et al., 2004; Reijrink et al., 2008; Salahi et al., 2012; Rocha et al., 2013). In this research, some of the results showed that the exposure of eggs to mechanical vibrations could also negatively affect the productive responses in hatchery facilities.

As a general rule, the treatment composed by greater intensity of vibration and greater exposure time (RSS $7.5 \mathrm{~m} \mathrm{~s}^{-2} ; 180 \mathrm{~min}$ ) was related to the worst results, in relation to the control. Similarly, Torma and Kovácsné (2012) proved that when hatching eggs are vibrated in an intense treatment $(30 \mathrm{~Hz} ; 10 \mathrm{~min})$, they present a

Table 3. Mean and standard deviation of high quality chicks hatched (in percentages), odds of a high quality chick, and odds ratios between the factorial treatments and control with $95 \%$ confidence intervals.

\begin{tabular}{lcccc}
\hline Treatments & High quality chicks (\%) & Odds of high quality chick & Odds ratios & Confidence intervals \\
Control* & $88.75+1.50$ & & & - \\
Block 1 & - & 16.96 & 1.00 & $(0.72 ; 1.93)$ \\
\hline Block 2 & - & 20.01 & 1.18 & $(0.38 ; 0.92)$ \\
Block 3 & - & 10.14 & 0.60 & $(0.58 ; 1.46)$ \\
\hline Block 4 & - & 15.60 & 0.92 & $(0.54 ; 1.73)$ \\
Factorial treatments & & & 0.96 & $(0.53 ; 1.79)$ \\
T1 & $89.25+2.87$ & 16.28 & 0.99 & $(0.33 ; 0.97)$ \\
T2 & $88.00+3.30$ & 16.79 & 0.57 & $(0.22 ; 0.59)$ \\
\hline T3 & $85.25+3.84$ & 9.66 & 0.36 & \\
T4 & $80.00+3.46$ & 6.10 & & \\
\hline
\end{tabular}

${ }^{*}$ Control treatment in block 1 was considered as the baseline for odds ratios comparisons; ${ }^{\circ}$ significant effects using the Wald test at $5 \%$ level of significance.

Table 4. Mean and standard deviation of the hatching egg losses (in percentages), odds of a hatching egg being lost, and odds ratios between the factorial treatments and control with $95 \%$ confidence intervals.

\begin{tabular}{|c|c|c|c|c|}
\hline Treatments & Hatching egg losses (\%) & Odds of losing a hatching egg & Odds ratio & Confidence intervals \\
\hline Control* & $6.0+2.16$ & & & \\
\hline Block 1 & - & 0.06 & 1.00 & - \\
\hline Block 2 & - & 0.05 & 0.85 & $(0.52 ; 1.38)$ \\
\hline Block $3^{\circ}$ & - & 0.10 & 1.67 & $(1.09 ; 2.59)$ \\
\hline Block 4 & - & 0.06 & 1.09 & $(0.68 ; 1.73)$ \\
\hline \multicolumn{5}{|c|}{ Factorial treatments } \\
\hline $\mathrm{T} 1$ & $6.5+2.88$ & 0.06 & 1.04 & $(0.58 ; 1.86)$ \\
\hline $\mathrm{T} 2$ & $6.5+1.73$ & 0.06 & 1.01 & $(0.56 ; 1.82)$ \\
\hline $\mathrm{T}^{\circ}$ & $10.0+3.39$ & 0.10 & 1.74 & $(1.03 ; 2.99)$ \\
\hline $\mathrm{T} 4^{\circ}$ & $14.75+2.34$ & 0.16 & 2.75 & $(1.69 ; 4.60)$ \\
\hline
\end{tabular}

*Control treatment in block 1 was considered as the baseline for odds ratios comparisons; ${ }^{\circ}$ significant effects using the Wald test at $5 \%$ level of significance. 
significant reduction in hatchability $(64.89 \%)$, in comparison to eggs exposed to intermediate vibrations of $20 \mathrm{~Hz}(76.80 \%)$ and in control group $(80.74 \%)$. These authors believe that prejudice to hatch, regarding to mechanical vibrations exposure, is a consequence of the alteration of the vibrated eggs' internal components.

A possible explanation to Torma and Kovácsné (2012) hypothesis, which is raised in this research, may be supported in Berardinelli et al. (2003a, 2003b) works. These authors verified that exposure of eggs to vibration effects turns the albumen's denser portion (intermediate) more liquid, which is confirmed by a significant reduction of up to $28 \%$ in Haugh units' average values. The albumen's liquefaction process is related to eggs' quality reduction and premature aging (Berardinelli et al., 2003a, 2003b).

It is known that the albumen is responsible for protecting the embryo of mechanical and microbiological agents, support nutrients and control the flow of water and gases with the environment. The albumen's quality is intimately associated with the liquefaction of its dense portion, which according to Scott and Silversides (2000), depends on both the factors pre-posture (chicken's age, strain, and nutrition), as well as, the time and the conditions of eggs storage. Reijrink et al. (2008) explain that when the albumen becomes very fluid, it facilitates the loss of water and oxygen in the egg to the external environment.

Torma and Kovácsné (2012) concluded that the reduction in hatchability for treatment with intense vibration $(30 \mathrm{~Hz})$ was due to a higher incidence of early mortality ( 1 to 6 day), with $19.52 \%$ of the total observations, in contrast with the results obtained in this research, since it is classification did not differ in any of the factorial treatments. It is believed that the results demonstrate that the reduction in hatchability of $\mathrm{T} 4$, is in part, explained by the increase of deaths in intermediate period ( $6^{\text {th }}$ to $17^{\text {th }}$ day), which was statistically higher than in the control.

For Tona et al. (2004), day-old chicks represent the end product of the incubator and the starting material for broiler farms. Ulmer-Franco et al. (2010) and Salahi et al. (2012) explain that the birds' quality is the result of all the events that occur with the hatching eggs, before and during embryonic development. Thus, results from the study may be regarded as further proofs for negative effects of high intensity mechanical vibration (RSS $7.5 \mathrm{~m} \mathrm{~s}^{-2}$ ).

The good quality chick is associated with the conditions pre and during incubation that affect the internal structure of the hatching egg and, consequently, the embryo's physiology, such as the efficiency of $\mathrm{CO}_{2}$ and $\mathrm{O}_{2}$ exchanges (Khabisi et al., 2012; Rocha et al., 2013). Thereby, it is the same argument presented for the hatchability response, which it is believed that the exposure of eggs to intense vibrations is related to changes in albumen and yolk, similarly to what occurs with the hatching eggs in long periods of storage and/or kept on inadequate environmental conditions.

The hatcheries reduce their profits when they pay for hatching eggs that break out, or which result in low quality chicks that will be discarded or sold for a lower value. Therefore, it can be stated that if a transport occurs with conditions similar to those simulated in the laboratory and with the vibration intensity resulting close to $7.5 \mathrm{~m} \mathrm{~s}^{-2}$, the hatching eggs losses at the end of the transport can be up to $10 \%$ higher than expected ( $6 \%$ in the control), which in economic terms is alarming. When these results are complemented with the increase of cracked and broken eggs described by Nazareno et al. (2013), whose tracks of vibration obtained in field are close to simulated, give weight to the inclusion of mechanical vibrations as a capable agent of increasing losses at hatch and decreasing the quality of broiler chicks.

\section{Conclusions}

The mechanical vibrations harmfully affect the hatch and the quality of broiler chicks. The combination of higher intensity with the longest exposure reduced hatchability and the proportion of high quality chicks. As a result, the total loss was higher, which shows a productive/economic prejudice of great relevance for poultry production.

\section{References}

Abeyesinghe S.M., Wathes C.M., Nicol C.J., Randall J.M. 2001. The aversion of broiler chickens to concurrent vibrational and thermal stressors. Appl. Animal Behav. Sci. 73:199-215.

Bailey R.A., Łacka A. 2015. Nested row-column designs for nearfactorial experiments with two treatment factors and one control treatment. J. Stat. Plann. Infer. 165:63-77.

Berardinelli A., Donati V., Giunchi A., Guarnieri A., Ragni L. 2003a. Effects of sinusoidal vibrations on quality indices of shell eggs. Biosyst. Eng. 86:347-53.

Berardinelli A., Donati V., Giunchi A., Guarnieri A., Ragni L. 2003b. Effects of transport vibrations on quality indices of shell eggs. Biosyst. Eng. 86:495-502.

Carlisle A.J., Mitchell M.A., Hunter R.R., Duggan J.A., Randall J.M. 1998. Physiological responses of broiler chickens to the vibrations experienced during road transportation. Br. Poultry Sci. 39:48-9.

Gebresenbet G., Van de Water G., Geers R. 2011. Vibration levels and frequencies on vehicle and animals during transport. Biosyst. Eng. 110:10-9.

Khabisi M., Salahi A., Mousavi S.N. 2012. The influence of eggshell crack types on hatchability and chick quality. Turk. J. Vet. Animal Sci. 36:289-95.

Nazareno A.C., Silva I.J.O., Vieira F.M.C., Santos R.F.S. 2015. Temperature mapping of trucks transporting fertile eggs and day-old chicks: efficiency and/or acclimatisation?. Rev. Brasil. Eng. Agr. Ambient. 19:134-9.

Nazareno A.C., Silva I.J.O., Vieira A.M.C., Vieira F.M.C., Miranda K.O.S. 2013. Níveis de vibração e choques em diferentes estradas durante o transporte de ovos férteis. Rev. Brasil. Eng. Agr. Ambient. 17:900-5.

R Development Core Team. 2005. R: a language and environment for statistical computing. R Foundation for Statistical Computing, Vienna, Austria. Available from: http://www.rproject.org/ Accessed: 09 January, 2013.

Randall J.M., Duggan J.A., Alami M.A., White R.P. 1997. Frequency weightings for the aversion of broiler chickens to horizontal and vertical vibration. J. Agr. Engine. Res. 68:387-97.

Randall J.M., Streader W.V., Meehan A.M. 1993. Vibration on poultry transporters. Br. Poultry Sci. 34:635-42.

Reijrink I.A.M., Meijerhof R., Kemp B., Van Brand H. 2008. The chicken embryo and its micro environment during egg storage and early incubation. World Poultry Sci. J. 64:581-98.

Rocha J.S.R., Baião N.C., Barbosa V.M., Pompeu M.A., Fernandes M.N.S., Lara L.J.C., Matias C.F.Q., Batista J.V.M.S.P. 2013. Negative effects of fertile egg storage on the egg and the embryo and suggested hatchery management to minimise such problems. World Poultry Sci. J. 69:79-89. 
Salahi A., Khabisi M., Pakdel A., Baghbanzadeh A. 2012. Effects of cold stress during transportation on hatchability and chick quality of broiler breeder eggs Ahmad. Turk. J. Vet. Animal Sci. 36:159-67.

Scott T.A., Silversides F.G. 2000. The effect of storage and strain of hen on egg quality. Poultry Sci. 79:1725-9.

Shannon S.G., Moran A.W., Mason K.T. 1994. Effect of vibration frequency and amplitude on developing chicken embryos. UAAARL Report 95:39-45.

Tona K., Onagbesan O., Ketelaere B., Decuypere E., Bruggeman V. 2004. Effects of age of broiler breeders and egg storage on egg quality, hatchability, chick quality, chick weight, and chick post-hatch growth to forty-two days. J. Appl. Poultry Res. 13:10-8.

Torma T., Kovácsné K.G. 2012. Effects of mechanical impacts on hatchability of broiler breeders. J. Agr. Sci. Technol. A 4:53540.

Ulmer-Franco A.M., Fasenko G.M., O'dea Christopher E.E. 2010. Hatching egg characteristics, chick quality, and broiler performance at 2 breeder flock ages and from 3 egg weights. Poultry Sci. 89:735-42. 